\title{
Entendiendo la Economía de la Salud: Una revisión de los estudios sobre eficiencia, equidad y desigualdades
}

\author{
Marta Pascual Sáez a , DAVId CANTARero Prieto ${ }^{\text {a }}$ \\ a Universidad de Cantabria, Facultad CC.EE., Avda. de los Castros, s/n., 39005 Santander, España. \\ E-mail:marta.pascual@unican.es, david.cantarero@unican.es
}

\begin{abstract}
RESUMEN
Este trabajo estudia el desarrollo e influencia actual de la economía de la salud en lo referente a los aspectos de eficiencia, equidad y desigualdades. Además, se muestra el estado actual del papel del sector público y privado así como su colaboración en la provisión y producción de servicios sanitarios. Los resultados obtenidos implican que la prospectiva de evolución de esta disciplina seguirá creando herramientas útiles en cuanto a ser aplicadas para conseguir mejorar la calidad de la atención sanitaria y la equidad en el acceso de sus ciudadanos. No obstante, para obtener una visión global en estudios de economía de la salud, es aconsejable utilizar varias medidas que se complementen entre sí.
\end{abstract}

Palabras claves: Economía de la Salud, eficiencia, equidad, desigualdades en salud, medición.

\section{Understanding Health Economics: A Review of Efficiency, Equity and Inequalities Studies}

\begin{abstract}
This paper examines the current development and influence of health economics to efficiency, equity and inequality issues. Besides, the current status of public and private sectors and their collaboration with health care provision and financing systems is showed. The results suggest that future evolution of health economics will create useful tools for improving quality and equity in access to health care services. Nevertheless, to obtain a comprehensive vision in relation with health economics studies, it is advisable to use a set of complementary measures.
\end{abstract}

Keywords: Health Economics, Efficiency, Equity, Inequalities in Health, Measurement.

JEL Classification: B20, I10

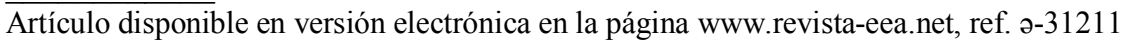




\section{INTRODUCCIÓN}

La Organización Mundial de la Salud (OMS) define la salud como "Un estado de completo bienestar físico, mental y social y no solamente la ausencia de afecciones o enfermedades". Esta definición (que no ha sido modificada desde $1948^{1}$ ) ha sido punto de partida desde hace décadas de numerosas investigaciones y estudios empíricos. Así, la OMS publica desde 1995, y de manera bianual, el "Informe sobre la salud en el mundo" que evalúa aspectos concretos relativos a la salud mundial como la financiación de los sistemas que la protegen, la atención primaria, el personal de dichos servicios sanitarios, las desigualdades en salud, prevención, tratamiento, atención y apoyo en el caso de enfermedad, salud mental, esperanza de vida, condiciones sanitarias, etc. Estos informes son un auténtico referente tanto para los países calificados como desarrollados como para los que están en vías de desarrollo.

A nivel europeo, el Convenio de Derechos Humanos de 1950 reconoce el derecho a la protección de la salud, siendo posteriormente reconocido y ratificado en la Carta Social Europa de 1962, en el Convenio para la protección de los derechos humanos y dignidad del ser humano (con respecto a las aplicaciones de la biología y medicina) de 1997 y en numerosas directivas y recomendaciones. Así, en el año 2000, se aprobó la Carta de Derechos Fundamentales en la Unión Europea que reconoce en su artículo 35 ese derecho a la protección de la salud de modo que: "Toda persona tiene derecho a la prevención sanitaria y a beneficiarse de la atención en ese sentido en las condiciones establecidas por las legislaciones y prácticas nacionales". Es decir, cada país, dentro de este marco general, diseña su propio marco normativo en materia de protección de la salud.

En nuestro país, no es casualidad entonces que en el artículo 43 de la Constitución de 1978 se reconozca el derecho a la protección de la salud y a la atención sanitaria de todos los ciudadanos. Y es la Ley General de Sanidad 14/1986, de 25 de abril, la que regula de modo general las acciones que permiten hacer efectivo este derecho aunque su desarrollo normativo posterior, con diferentes leyes y decretos ${ }^{2}$, no ha resuelto aún a nuestro entender todos los problemas de financiación, eficiencia y equidad inherentes a nuestro Sistema Nacional de Salud.

\footnotetext{
${ }^{1}$ Véase el Preámbulo de la Constitución de la OMS firmada el 22 de Julio de 1946 por representantes de 61 Estados y que entró en vigor el 7 de Abril de 1948.

${ }^{2}$ Como por ejemplo, la Ley 41/2002 de 14 de Noviembre, básica reguladora de la autonomía del paciente y de derechos y obligaciones en materia de información y documentación clínica; los correspondientes Reales Decretos que trasladan las competencias sanitarias a las Comunidades Autónomas (R.D. 1587/1981; R.D. 1472/2001; R.D. 1473/2001; R.D. 1474/2001; R.D. 1475/ 2001, R.D. 1477/2001; R.D. 1478/2001; R.D. 1479/2001; R.D. 1480/2001) o la Ley 16/2003, de 28 de Mayo, de cohesión y calidad del Sistema Nacional de Salud.
} 
En este contexto internacional y nacional y en paralelo al desarrollo normativo, la Economía de la Salud (Health Economics) surgió como disciplina autónoma dentro de la literatura económica en la década de 1960. El motivo principal de su creación fue la propia revisión del papel tan destacado tanto en términos cuantitativos como cualitativos que había alcanzado la sanidad dentro de los presupuestos de los diferentes Estados. La importancia creciente de este tipo de gasto social ha planteado así constantemente la necesidad de evaluar su grado de eficacia, eficiencia y equidad frente a la necesidad de invertir en otro tipo de gastos públicos. Dada la conjunción de diversos factores que presionan al alza dichos gastos sanitarios y la dificultad de controlar por parte de los diferentes sectores públicos a muchos de los factores determinantes del nivel de salud de los individuos (tales como los de tipo biológicos, medio ambientales o de estilos de vida), cada vez se ha vuelto más importante supervisar la necesidad de realizar inversiones en dichos servicios sanitarios más allá de sus componentes asistenciales. Son sus dimensiones económicas, políticas y sociales las que son fundamentales pues, para garantizar la igualdad de oportunidades en cualquier país.

Y es que si se comparan los sistemas sociales de provisión sanitaria con los de otro tipo de gastos sociales como la educación o la vivienda, puede observarse que en sanidad el grado de intervención pública alcanza más de tres cuartes partes del total. No obstante, la mayoría de bienes y servicios que constituyen el "gasto en sanidad" son privados, ya que en ellos su consumo es rival (beneficios divisibles) y se les aplica el principio de exclusión (técnicamente se les puede aplicar un precio unitario, excepto a partidas específicas como las relativas a la investigación o Salud pública). Eso configura desde un punto de vista teórico a la sanidad como un bien "comercializable" que podría ser producido privadamente aunque en la mayoría de países desarrollados se financia (provisiona) y produce por el sector público.

Tal es así que la Salud y su relación con la Economía ha venido siendo en las últimas décadas objeto de interés y estudio por parte de muchos economistas (y otros que no lo son estrictamente). La Economía de la Salud, como rama específica dentro de la literatura económica, tiene cada vez mayor influencia académica y social (Wagstaff y Culyer, 2012; Zweifel, 2013). Esto último puede medirse en términos bibliométricos recientemente por el índice- $h$ (o medida de productividad científica que hace balance entre el número de publicaciones y las citas que suscitan; y, por ejemplo, un índice de 4 implica que un autor tiene 4 publicaciones que han recibido al menos 4 citas cada una). Según dicho índice$h$, en el Top de los 100 economistas de la salud más influyentes en el período que va desde 1969 hasta nuestros días destacan, entre otros, Chaloupka, Currie, Cutler, Grossman, Gruber, Newhouse, Pauly, Sloan, Van Doorslaer, Viscusi, Wagstaff, Williams o Zweifel. 
Como decimos, dicha producción científica ha sido abundante especialmente en los últimos tiempos a través de diversas publicaciones en revistas académicas incluidas en el ISI-JCR Web of Knowledge y reuniones científicas tanto fuera de nuestras fronteras (International Health Economics Association Anual Meeting, Health Economics, Journal of Health Economics, Value of Health, Pharmacoeconomics, International Journal of Health care and finance, Health Policy, Social Science and Medicine, entre otras) como dentro de ellas (Jornadas de la Asociación de Economía de la Salud, de la Sociedad Española de Salud Pública y Administración Sanitaria, Congreso Nacional de Hospitales, Congreso Internacional de Economía Aplicada-ASEPELT, Gaceta Sanitaria, Hacienda Pública Española, Estudios de Economía Aplicada o Gestión Clínica y Sanitaria, entre otras). En todos esos ámbitos se han estudiado diversas cuestiones en el marco de un análisis económico específico que se diferencia del general. Y ello se debe a que en la sanidad, el "mercado" se caracteriza porque la "demanda está inducida por la oferta", lo que afecta al comportamiento de los agentes que lo componen (Phelps, 1986; Labelle et al., 1994). Por tanto, se diferencia del resto de literatura económica en cuanto a su presente y futuro, en la complejidad de preguntas que intenta contestar y los métodos empleados para ello (Fuchs, 2000; Zweifel, 2013).

Teniendo en cuenta estas premisas, la estructura de este artículo es la siguiente. En la Sección 2 se describe el estado actual del papel del sector público y privado así como su colaboración en la provisión y producción de servicios sanitarios. En la Sección 3 se presenta una revisión de la literatura en Economía de la Salud en cuanto a estudios que evalúan la eficiencia y equidad de los servicios sanitarios así como las desigualdades de salud existentes en cada país. Finalmente, se presenta una síntesis de resultados de dichos análisis así como una prospectiva sobre qué puede esperarse de la evolución de esta disciplina en el futuro.

\section{EL PAPEL DE LOS SECTORES PÚBLICOS Y PRIVADO EN SANIDAD}

Las actuaciones de las Administraciones Públicas Sanitarias están orientadas en general, y en España en particular (artículo 6 de la Ley General de Sanidad 14/1986), a la promoción de la salud, a promover el interés individual, familiar y social por ella mediante la adecuada educación sanitaria de la población, a garantizar que cuantas acciones sanitarias se desarrollen estén dirigidas a la prevención de las enfermedades (y no sólo a la curación de las mismas), a garantizar la asistencia sanitaria en todos los casos de pérdida de la salud y a promover las acciones necesarias para la rehabilitación funcional y reinserción social del paciente.

Sin embargo, en el "mercado" sanitario pueden producirse diversos fallos 
que desaconsejarían una provisión privada completa de servicios de salud (Cantarero, 2003). Entre otros, podríamos citar a los siguientes:

\section{a) Salud como Bien público y Externalidades}

En el consumo de asistencia sanitaria es posible que se generen externalidades (con beneficio más allá de la persona que recibe el servicio y mejoras en la productividad global) como: la erradicación de ciertas enfermedades (Externalidades tangibles); que el consumo sanitario de los demás incrementase la propia utilidad aunque eso no tuviese consecuencias directas para la salud (Externalidades filantrópicas o altruistas); que la posibilidad de acceso al servicio sanitario fuese beneficiosa, ya que sino las fuertes externalidades negativas afectarían a la tasa de crecimiento, en forma de individuos "productivos" contagiados por enfermedades y/o afectados (Externalidades "opcionales"); que el comportamiento de una persona afectase a la salud de otra debido al consumo de alcohol, tabaco y drogas ilegales además de estilos de vida perniciosos o Bienes perjudiciales (Externalidades negativas, que deben ser asumidas por el sistema sanitario tanto en tratamientos para corregir sus efectos nocivos sobre la salud como de políticas de prevención de su consumo).

\section{b) Rendimientos crecientes (o costes decrecientes) a escala o "monopolios naturales"}

Su existencia en dicho "mercado" sanitario justificarían su intervención por el sector público. Así, en muchas áreas rurales la baja densidad de población podría convertir en óptimo no dar cabida a más de un proveedor, pese a que crease situaciones de monopolio debidas a factores tecnológicos ("monopolios naturales") con el riesgo de que determinados agentes no pudiesen proveer los servicios eficientemente porque no alcanzarían el tamaño mínimo (debido a costes fijos elevados) para aprovechar las economías de escala.

\section{c) Incertidumbre e información asimétrica (Riesgo moral y Selección adversa)}

Newhouse (1970) y Grossman (1972) indicaban que son dos problemas derivados de la Teoría relativa a la "relación de agencia". En primer lugar, la propia Incertidumbre es inherente a todo el sector sanitario desde la incidencia de la enfermedad hasta el conocimiento de la eficacia del tratamiento médico.

En cuanto a la Información asimétrica como decíamos puede conllevar problemas derivados del riesgo moral y de la selección adversa. Así, el Riesgo moral se produce cuando el aseguramiento transforma una transacción bilateral (entre proveedor asistencial y paciente) en una trilateral (entre aseguradora, proveedor y paciente). Ello supone que el paciente se enfrenta a un precio "cero" al usar los servicios asistenciales consumiendo servicios sanitarios en la mayoría de los casos por encima de la cantidad deseada en caso de no estar 
asegurado por dicho riesgo (Beck et al., 2010). Como resultado, el proveedor siempre tenderá a explotar la diferencia en términos de información por lo que puede no existir una demanda independiente de la oferta de servicios sanitarios y los mecanismos de mercado no podrían operar (al menos, para cierto segmento de la oferta), dados los problemas de la "demanda inducida por la oferta" (Phelps, 1986). Asimismo, es posible que los individuos con seguro médico tiendan a "sobre utilizar" los servicios sanitarios más del nivel óptimo o "socialmente" deseable, debido a que poseen también información adicional sobre la verdadera intensidad de sus molestias y sufrimientos difícilmente verificable por el facultativo y que están, sin embargo, cubiertas por el seguro sanitario.

En cambio, la Selección adversa se produce cuando se oculta información por parte del paciente sobre una situación de riesgo, lo que haría al "mercado" sanitario incompleto y proporcionaría un nivel de cobertura (en cuanto a aseguramiento) menor al "socialmente" deseable. Ello obligaría a todos los sujetos a pertenecer al esquema de seguro (no necesariamente con gratuidad universal) y que estas deficiencias se resolviesen mediante intervenciones (barreras de entrada) con la creación de poder de mercado que supone ocultar información sobre una situación de riesgo (regulación sobre certificación, acreditación, práctica médica o fármacos). Todo esto haría difícil la entrada y salida de los "mercados" sanitarios alejándoles del comportamiento de los "mercados perfectos" y por eso sólo ofreciendo a todos los individuos una única prima de riesgos promedio, llegaría a configurarse un mercado completo de seguros, aunque ineficiente (Stiglitz, 1994).

¿Justifican entonces todos los "fallos de mercado" anteriormente señalados la intervención pública total en el "mercado" sanitario? Para algunos sí. Y además le unen argumentos como la posible influencia del gasto sanitario en el crecimiento económico (aunque resultaría difícil aislar su efecto del de otros gastos sociales) así como dos principios básicos: el paternalismo del Estado (como agente o juez mejor informado de qué es lo que conviene a los individuos, lo que le llevaría a prohibir el consumo de ciertos bienes "indeseables") y que la sanidad sea un "Bien preferente" y un derecho ya que su consumo se asumiría como necesario (al menos, hasta ciertos niveles mínimos), con independencia de las preferencias individuales o principio ético redistributivo (que nadie acceda preferentemente a los elementos esenciales para conservar la vida por el hecho de tener capacidad para poder comprarlos y no pueda sacar provecho de la venta de su propio cuerpo o de órganos o sangre del mismo). Esto último justificaría intervenciones públicas como la cobertura médica pública y la prohibición de "comercialización" de sangre y órganos (Cabasés y Errea, 2010).

De todos modos, no basta con la simple presencia del sector público en 
sanidad para garantizar plenamente todos los objetivos que justificaban dicha intervención. Y eso porque su respuesta, aún siendo bien intencionada, puede resultar finalmente perjudicial debido a los propios "fallos inherentes al funcionamiento del sector público" debidos a:

\section{a) Planificación burocrática y acción política}

En este caso, las "asimetrías" de información entre quienes aprueban los programas de gasto público (parlamentos y acción política en sistemas democráticos como los nuestros) y los que los ejecutan (agencias planificadoras burocráticas) pueden incentivar a que éstas últimas, al disfrutar de una posición cuasi monopolística, pudiesen "aprovechar o explotar" la información de la que disponen en su propio beneficio (positivamente correlacionado con el presupuesto que gestionan) lo que llevaría a una sobre expansión del mismo por encima del nivel considerado como socialmente óptimo. Como resultado, sería posible que las ganancias potenciales en eficiencia, que una intervención pública en sanidad debiera aportar, se vieran anuladas por una pérdida de bienestar social mayor.

Además, si hipotéticamente el Estado demostrara su firmeza en el compromiso de no ayudar a aquellos que no pudiesen pagarse su atención sanitaria, todos los individuos asumirían la responsabilidad personal del mantenimiento y protección de su propia salud asegurándola de algún modo en el mercado, que alcanzaría así la eficiencia ("mano invisible"). Pero, la incapacidad de los actuales Estados del Bienestar para mantenerse firmes en ese compromiso de no ayuda debido a razones de equidad y justicia social, es la que causaría el fallo (o ausencia) de mercados completos de salud privados, encontrándose así soporte a la existencia de un sistema de seguro sanitario obligatorio (aunque no necesariamente público).

\section{b) Ineficiencias e inequidades}

Aunque un asegurador público resolviese los numerosos problemas de un "mercado sanitario", (como selección adversa o externalidades), ello no le eximiría de fallos como el Riesgo moral y la "demanda inducida por la oferta" y que comparte con el propio sector privado. Además, ningún sistema sanitario hasta la fecha ha sido capaz de medir de forma eficiente la cantidad y calidad de asistencia prestada por los diferentes centros por lo que al sector público, entidades aseguradoras, familias o empresas les es difícil controlar el gasto sin afectar a su calidad . Y tampoco la equidad en el reparto del gasto sanitario está totalmente garantizada por el hecho de estar financiado mayoritariamente por el sector público, puesto que un sistema caracterizado por gratuidad en sus prestaciones y racionamiento basado en los tiempos de espera puede no asegurar la equidad en el acceso y utilización de asistencia sanitaria. 


\section{UNA REVISION DE LA LITERATURA EN ESTUDIOS DE ECONOMÍA DE LA SALUD: EFICIENCIA, EQUIDAD Y DESIGUALDADES}

Una de las bases fundamentales de la Economía (y de la Economía de la Salud) es la Eficiencia entendida en términos globales como aquella situación en la que en una determinada asignación de recursos hace que el nivel de utilidad (o producción según se entienda) no pueda mejorar a no ser que el de otra empeore (es decir, conseguir lo máximo a partir de recursos escasos). En las siguientes páginas se repasan los principales estudios en economía de la salud en dicha línea. Dado que toda decisión implica también una renuncia, la sociedad se enfrenta así a una disyuntiva importante: ¿mayor eficiencia puede conseguirse sin menoscabar la equidad?

Y es que la Equidad trata de distribuir la riqueza de forma "justa" (según lo que se entienda por tal en la función de bienestar social) entre los miembros de una sociedad. Obviamente, el propio reconocimiento de que dichos individuos se enfrentan a disyuntivas no nos indica por sí solo qué decisiones deberían tomar pero sí sirve para entender cuáles son las opciones que tienen. Por ello, la relación de intercambio o trade off entre más eficiencia sin perjudicar la equidad no resulta sencilla para los gobiernos a la hora de diseñar su política sanitaria.

El objetivo de equidad en el caso de la salud se puede intentar conseguir tanto a través de la política de ingresos como de gastos públicos, o mediante una combinación de ambas. Alcanzar la equidad al financiar la sanidad exclusivamente por medio de ingresos públicos, con tipos elevados, puede aportar suficiencia al sistema pero a su vez generar altos costes de eficiencia al incrementarse el exceso de gravamen (o coste de los impuestos en la sociedad en términos de bienestar social). Por eso, aquellos gobiernos dispuestos a sacrificar eficiencia a favor de una mejor distribución de la renta (en términos de equidad) optarán por sistemas impositivos más progresivos en cuanto a la financiación de la sanidad, mientras que aquéllos que prefieran una mayor eficiencia o neutralidad lo harán por impuestos y tipos más reducidos, dando un mayor protagonismo al gasto público en sanidad. Es decir, la equidad en sí misma es un concepto de difícil valoración, pues puede referirse a un momento concreto del tiempo o a un período más flexible, con el inconveniente adicional que gastos como las inversiones en sanidad como hospitales, centros de salud o tecnología y equipamiento suelen proyectarse en horizontes plurianuales tan extensos que dificultan valorar todos sus efectos.

Por tanto, en las siguientes líneas se revisarán (además de para el caso de la eficiencia) también los principales estudios relativos a la equidad y desigualdades en salud entendidas como la búsqueda de situaciones de justicia social e igualdad en condiciones de oportunidad y acceso y utilización a los servicios de asistencia sanitaria. 


\subsection{Eficiencia en Economía de la Salud}

Seminalmente fue definida por Farrell (1957) y puede entenderse de dos maneras. En cuanto a la primera, alcanzar la eficiencia técnica supone alcanzar la cantidad máxima de producción a partir de una cantidad dada de inputs o también producir un nivel concreto de output utilizando la mínima cantidad de recursos posible. La segunda acepción de eficiencia se refiere a la eficiencia asignativa que se cumple cuando la combinación de inputs logra minimizar los costes dados sus precios o bien cuando la producción permite maximizar los ingresos dados los precios de venta. Ambos tipos de eficiencia se engloban dentro del concepto de eficiencia global.

En el caso concreto que nos ocupa referente a los sistemas sanitarios, la eficiencia global precisamente se conseguiría al alcanzar el mejor estado de salud posible de una población dada una cantidad de gasto público sanitario invertido. El resultado relevante se mide pues en indicadores de resultado final (output) que en este caso podrían ser muertes evitables, esperanza de vida, tasas de mortalidad infantil o Años de Vida Ajustados por Calidad (AVACs).

Figura 1

La eficiencia y su evaluación en síntesis

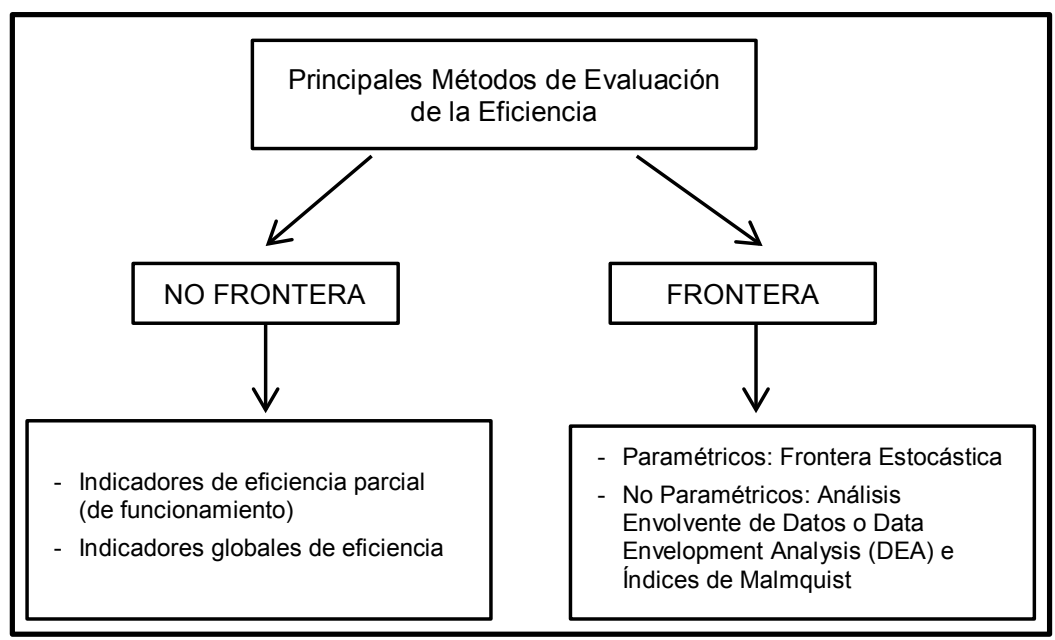

Fuente: Elaboración propia.

La principal cuestión para medir dicha eficiencia global es si se adopta el enfoque de evaluar cuánto de cerca está una organización sanitaria de la frontera de mejor práctica. Este modo medir la eficiencia (a diferencia del análisis no frontera, con un marco teórico menos formalizado) así como la evaluación comparativa (benchmarking) del rendimiento se fundamenta en las informacio- 
nes sobre costes, productos y recursos para imputar un índice de eficiencia relativa en cuanto a la mejor práctica dentro de la muestra de entidades sanitarias que se analicen (Puig-Junoy y Dalmau, 2000; Martin y Puerto, 2007). En la literatura, al evaluar la eficiencia relativa se han utilizado fundamentalmente (Figura 1) estos métodos: no frontera (con indicadores de eficiencia parcial e indicadores globales de eficiencia); fronteras paramétricas (como la frontera estocástica) y fronteras no paramétricas (análisis envolvente de datos e índices de Malmquist).

En cuanto a la aproximación No Frontera son múltiples los indicadores de eficiencia parcial (pues relacionan un solo output con un solo input) de funcionamiento (performance indicators) en función de calidad, resultados en salud, productividad, etc. En cuanto a los indicadores globales de eficiencia si se agrupan por áreas homogéneas da lugar al Cuadro de Mando (para el ámbito financiero y de actividad), que si se completa con factores estratégicos como satisfacción de clientes, procedimientos internos, desarrollo de la empresa, o innovación configura un Cuadro de Mando Integral (Balance Scorecard). Asimismo, también dentro de dichos indicadores globales podemos encontrar a los perfiles de proveedores (profiling) que sirven para comparar transversalmente proveedores sanitarios en función de indicadores epidemiológicos, asistenciales o de resultados en salud. Finalmente, el Benchmarking identifica los mejores centros sanitarios y sus prácticas, fijando estándares (por ejemplo, el coste medio por proceso) y comportamientos estratégicos para que todas las unidades evaluadas mediante esta técnica puedan ir acercándose al nivel de dichos "mejores" centros.

Es importante señalar que aunque el análisis no frontera parece ser bastante gerencialista y menos académico (que el enfoque frontera) tiene como inconveniente la falta de un marco teórico que defina claramente el concepto de eficiencia pudiendo existir contradicciones entre diferentes ratios o índices (Peiró, 2006).

En lo referente a la segunda aproximación que es la de Frontera puede a su vez dividirse en fronteras paramétricas y no paramétricas que difieren en el tipo de medida que calculan, los datos que precisan, y los supuestos que conllevan respecto de la estructura de la tecnología productiva. La cuestión clave aquí es que una forma paramétrica es más restrictiva que una forma no paramétrica.

En estos casos, los dos métodos más utilizados en la literatura son el enfoque de la frontera estocástica dentro de los métodos frontera paramétricos y el análisis envolvente de datos o Data Envelopment Analysis (DEA), dentro de los métodos frontera no paramétricos, los cuales precisan de métodos econométricos y programación lineal, respectivamente. El primer enfoque relativo a la frontera paramétrica estocástica es el que implica que los costes observados (o la producción) de una Unidad de Toma de Decisiones se pueden desviar de la 
frontera de costes o de la de producción o bien por fluctuaciones aleatorias o por ineficiencia.

El segundo enfoque relativo a técnicas no paramétricas engloba, entre otros, al DEA e índices de Malmquist como técnicas deterministas. En cuanto al DEA, supone que todas las desviaciones entre costes o producción observada y el coste mínimo o producción máxima en la frontera se deben a comportamientos ineficientes (para una buena revisión detallada puede consultarse a Lovell, 1993; Coelli et al., 1998; López-Casasnovas y Wagstaff, 1988; Hollingsworth, 2008). Dicho DEA se configura pues como una técnica de programación matemática que compara la eficiencia técnica de organizaciones (Decision Making Units - DMU o Unidades de Toma de Decisiones) que funcionan en contextos similares y tienen inputs y outputs multidimensionales. Así, una entidad sanitaria sería eficiente si no hay otra entidad o combinación lineal de ellas que pueda mejorar alguno de sus outputs sin empeorar al mismo tiempo alguno de sus otros outputs (DEA orientado a output)o inputs (DEA orientado a input)

Asimismo, como otra técnica determinista el cálculo de índices de Malmquist es uno de los métodos más comúnmente utilizados para analizar la evolución de la productividad y sus componentes en el tiempo.

Como ventajas cabe destacar que no precisa realizar supuestos sobre la forma funcional de la función de producción al ser un método no paramétrico y permite descomponer la productividad total de los factores de una unidad productiva en el cambio debido a la mejora de la eficiencia técnica (y ésta a su vez en eficiencia pura y eficiencia de escala) y el debido al progreso tecnológico.

A su vez, estos índices de Malmquist muestran el cambio en la eficiencia técnica y el cambio tecnológico, valorando si las $D M U$ se acercan o no a su correspondiente frontera de eficiencia entre dos períodos además de medir el movimiento de la frontera para conocer si las unidades que forman la frontera de eficiencia han mejorado o no su productividad entre los períodos analizados. Por todo ello estos índices de Malmquist han sido extensamente utilizados en el sector público y para el caso sanitario.

Sin ánimo de ser exhaustivos, en un repaso a la literatura sobre esta temática cabe indicar que los estudios acerca de la medida de la eficiencia de las organizaciones sanitarias en España tienen sus orígenes en el artículo publicado en Applied Economics por Wagstaff (1989) donde estimaba mediante técnicas paramétricas la eficiencia de una muestra de hospitales del Insalud mientras que el artículo de Ley (1991) en Investigaciones Económicas supone la primera aplicación del DEA a hospitales españoles.

Más en general, Hollingsworth y Street (2006) indican que, a pesar de que la investigación aplicada en eficiencia para sanidad ha sido intensa en los últimos tiempos, sigue existiendo una importante diferencia entre el enfoque de la oferta y el de la demanda decantándose desde mediados de los 80 en el caso de la 
Economía de la Salud por el hecho de que son muchos más los trabajos realizados desde la perspectiva de la oferta que desde la demanda (recordemos como señalábamos anteriormente que en el "mercado" sanitario la demanda está inducida por la oferta).

A pesar de la amplia difusión posterior de estudios de medida de la eficiencia en las organizaciones sanitarias que han seguido a todos los mencionados líneas atrás, siguen existiendo muchos problemas que limitan estas investigaciones. Y eso debido a que los índices de eficiencia técnica o económica no se pueden interpretar directamente como el resultado de una buena gestión sanitaria en la medida en que se producen también por recursos omitidos, diferencias en la calidad de los recursos y del producto, en la tecnología productiva o en la propia selección de variables ya utilizadas en la literatura existente en el área de la eficiencia en sanidad (Hua et al., 2009; Kao et al., 2011; Leleu et al., 2012; Puig-Junoy, 2000).

A eso se une que elaborar una lista ordenada (ranking) de agentes proveedores de servicios sanitarios tratando de mejorar su efectividad y eficiencia podría tener repercusiones importantes en términos de financiación y gestión que habría de manejarse con suma precaución (Murillo y González, 2006)

\subsection{Equidad y desigualdades en Economía de la Salud}

La definición de equidad en salud es compleja y no está exenta de juicios de valor que como tales son cambiantes con el tiempo. De manera general, hace referencia a la ausencia de diferencias en salud evitables, injustas o modificables entre grupos de personas y está intrínsecamente unido a los conceptos de desigualdad, justicia distributiva y justicia social (véase World Health Organization, 2009). Así, el análisis de las desigualdades en salud y sus factores determinantes sigue siendo un objetivo prioritario de los modernos Estados de Bienestar.

Es evidente el interés de cualquier país o sociedad por el estudio de la desigualdad y las implicaciones que ello supone. Trabajos como los de Gini (1921), Kolm (1968 y 1976), Atkinson (1970), Sen (1973 y 1992), Jakobsson (1976) y Kakwani (1977) han servido de referencia en numerosos estudios empíricos posteriores. Sin embargo la posible introducción de juicios de valor puede ocasionar problemas. Y adicionalmente, se plantean el problema de no comparabilidad de dos distribuciones de salud. Este inconveniente se resuelve, en muchas ocasiones, con la introducción de numerosas medidas de desigualdad que permiten establecer órdenes completos, frente a los órdenes parciales que proporcionan las curvas de Lorenz o las curvas de concentración.

El análisis de las desigualdades en salud ha sido abordado desde diferentes perspectivas. Por una parte, podríamos destacar los métodos basados en curvas e índices de concentración de salud y curvas de Lorenz como medidas de de- 
sigualdad relativa en salud relacionada con la renta (Wagstaff et. al., 1989; Wagstaff y van Doorslaer, 2000). De manera general, el índice de concentración puede expresarse como:

$$
C=\frac{2}{\mu} \sum_{i=1}^{N}\left(y_{i}-\mu\right)\left(R_{i}-\frac{1}{2}\right)=\frac{2}{\mu} \operatorname{cov}\left(y_{i}, R_{i}\right),
$$

donde $y_{i}$ es la salud del individuo $i, R$ es el ranking o posición relativa del individuo $i$ en la distribución de la renta y $\mu$ es la media de salud en la población.

Una ventaja adicional se basa en el hecho de que muchos de los índices propuestos pueden descomponerse en factores (Kakwani, 1980) que permiten profundizar aún más en las diferencias en las desigualdades en salud. En este sentido, son numerosos los estudios realizados que ponen de manifiesto las existentes desigualdades en salud por grupos sociales, según género y por grupo de edad, etc. (Thouez, 2006; Stoyanova et al., 2008). Para ello, metodológicamente, la utilización de modelos probit o logit ordenados así como la regresión por intervalos se convierten en herramientas fundamentales.

En todos estos estudios citados anteriormente es habitual utilizar, entre otras, como medidas de salud, la autovaloración o estado de salud percibido, estadísticas de mortalidad, esperanza de vida al nacer y supervivencia, indicadores de morbilidad basados en la incidencia y prevalencia de enfermedades de acuerdo a diagnósticos médicos, la presencia de al menos una enfermedad crónica y las limitaciones para realizar la actividad diaria. Además, la autovaloración que los individuos hacen de su propio estado de salud se considera que tiene un fuerte poder predictor de otros indicadores de carácter objetivo como la esperanza de vida, la mortalidad y la utilización de servicios sanitarios (Van Doorslaer y Gerdtham, 2003). En todos estos estudios se confirma la existencia de una cierta asociación entre nivel de renta o nivel socioeconómico y salud.

Una vez constatada la existencia de desigualdades en salud, el objeto de interés se traslada a la búsqueda de las causas o factores que determinan las mismas (Mackenbach, 2005). Así, además de la renta, las características sociodemográficas (sexo, edad, lugar de residencia, situación laboral, etc.), los estilos de vida (consumo de tabaco y alcohol, ejercicio físico, alimentación, etc.) y los factores psicológicos (estrés, situación emocional, etc.) son considerados piezas clave en estos estudios (Wilkinson, 1997). Todos estos factores podrían explicar asimismo las desigualdades en morbilidad y las diferencias en utilización sanitaria (Costa y Gil, 2008). Incluso han surgido estudios cuyo objetivo es analizar la transmisión intergeneracional de salud (Rivera et al., 2008; Pascual y Cantarero, 2009). Por otra parte, y más recientemente, se han desarrollado numerosos estudios cuyo objetivo es la evaluación de los distintos programas y en especial con aquellos que persiguen reducir las situaciones de equidad y desigualdad en términos de salud. Así, el desarrollo de técnicas cuasi-experimentales (entre las 
que se encuentra el Propensity Score Matching) ha sido clave (Rosemnbaum y Rubin, 1983; Abadie e Imbens, 2006; Heckman et al., 1998; Heckman, 2001; Imbens y Wooldridge, 2009). En estas investigaciones se utilizan fundamentalmente como indicadores de salud información relativa a mortalidad, esperanza de vida al nacer y supervivencia, indicadores de salud percibida o de morbilidad basados en la incidencia y prevalencia de enfermedades de acuerdo a diagnósticos médicos.

Además, cabe señalar que el desarrollo de todos estos estudios empíricos se ha visto fuertemente fortalecido no sólo por los últimos avances estadísticos y econométricos sino también por una mayor disponibilidad de fuentes de información aplicables a estudios de equidad y desigualdades en salud. En ese sentido, y al igual que sucede de modo similar en otros países, en España, el Instituto Nacional de Estadística (INE) ofrece gran cantidad de información relacionada con la economía, la demografía y la sociedad en general. Así, la publicación periódica por parte del INE de estadísticas oficiales permite un mejor conocimiento de las características socio-demográficas de la población. En particular, en el ámbito de la Salud (pero también de la dependencia y servicios sociales), la publicación de encuestas periódicas ha permitido conocer mejor no sólo el estado de salud de la población sino también los factores determinantes de la misma, causas de defunción, morbilidad, número de profesionales sanitarios colegiados en España, prevalencia y situación de las personas que sufren algún tipo de discapacidad, etc.

De este modo y a modo de resumen, podemos señalar un conjunto de variables que sin duda permiten a los investigadores en esta temática profundizar en el estudio de las desigualdades en salud como son estado de salud percibido, número de visitas al médico general o especialista, tasas de hospitalización, mortalidad y esperanza de vida aunque sin duda estos estudios se complementan con el análisis no sólo de las características básicas de los individuos sino también con dos variables en sanidad muy relevantes como son su gasto y financiación. Dichas variables habrían de garantizar no sólo la equidad a nivel nacional sino también interregional permitiendo así realizar análisis de equidad en el acceso al servicio sanitario público tanto desde el punto de vida de equidad horizontal como vertical y de igualdad de oportunidades (Montero y Jiménez, 2008).

La Encuesta Nacional de Salud se ha convertido con el paso del tiempo en un auténtico referente tanto a nivel nacional como por Comunidades Autónomas. El objetivo de esta encuesta es obtener datos sobre el estado de salud en nuestro país así como los determinantes personales, sociales y ambientales que determinan la salud y el uso de los servicios sanitarios. Los últimos datos disponibles han sido recientemente publicados (Abril 2013) y se corresponden a entrevistas realizadas entre julio de 2011 y junio de 2012 de ahí que nos refiramos 
a ella como ENSE 2011-12. Las inmediatamente anteriores publicaciones de la Encuesta Nacional de Salud (ENS) como bien se señala en www.ine.es son las correspondiente a 2003 y 2006.

A modo de ejemplo con este tipo de Encuestas la Figura 2 recoge por Comunidades Autónomas dos indicadores de salud tradicionalmente empleados en estudios de Economía de la salud como son la autovaloración que los individuos hacen de su propio estado de salud y la esperanza de vida al nacer. Un análisis visual y descriptivo, pero muy intuitivo, nos permite detectar que hay dos regiones (Madrid y Navarra) con el mayor porcentaje de población con salud percibida buena o muy buena y la mayor esperanza de vida al nacer. Profundizar en los aspectos que justifican y explican estas desigualdades territoriales es objeto de estudio en el marco de Economía de la Salud.

Figura 2

Indicadores de Salud en España: Autovaloración en salud versus Esperanza de Vida al Nacer

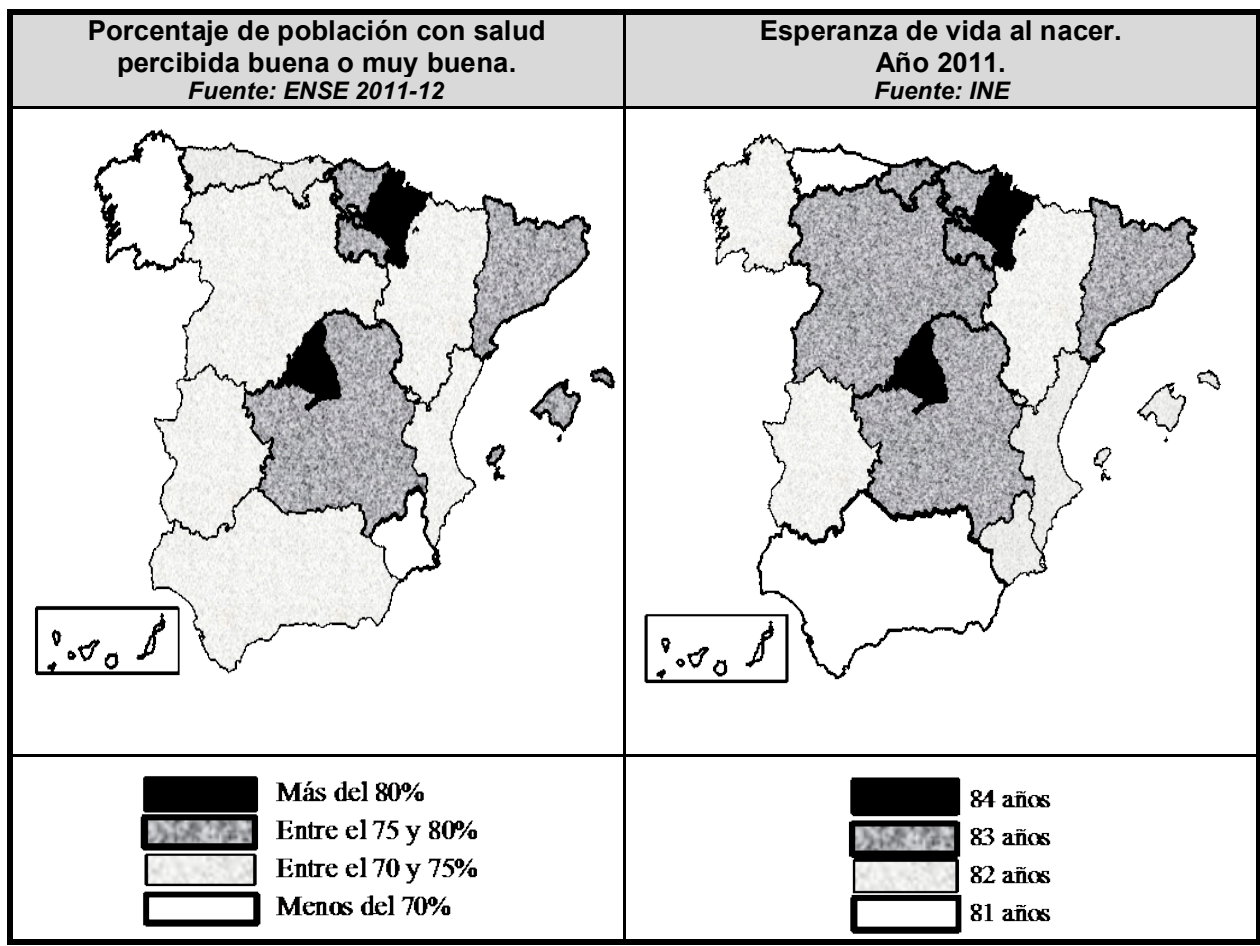

Fuente: Elaboración propia.

Por otro lado, a nivel europeo la evidencia empírica se basa fundamentalmente en el correspondiente módulo de salud de las estadísticas armonizadas 
para los países de la Unión: a saber, la Encuesta de Condiciones de Vida (20042012) y el Panel de Hogares de la Unión Europea (1994-2001). Esta información se complementa con las estadísticas publicadas por la Organización Mundial de la Salud y la Organización para la Cooperación y Desarrollo Económico.

Por otra parte, los estudios realizados podrían clasificarse, de manera general, según el tipo de análisis (descriptivos y analíticos), en función del momento del tiempo en el que se registran las observaciones (transversales y longitudinales), en función del momento temporal del suceso con respecto a la posición adoptada por los investigadores (prospectivo o retrospectivo), en función de la intervención de los investigadores (observacionales y experimentales) y en función de la unidad de análisis del estudio (individuales y ecológicos).

\subsection{Una aproximación al impacto bibliométrico de los estudios de eficiencia, equidad y desigualdades en Economía de la Salud}

Un elemento adicional y que constata la relevancia de los temas anteriormente planteados, es el impacto alcanzado por las correspondientes publicaciones científicas. En ese sentido y a la vista de revisiones similares a ésta (Rubin y Chang, 2003; Wagstaff y Culyer, 2012), teniendo en cuenta a la hora de buscar el impacto bibliométrico de los principales estudios de eficiencia y equidad o Top 10 de publicaciones científicas (véase Tabla 1), se ha consultado EconLit (http://www.aeaweb.org/econlit/doctypes.php) que además incluye working papers a través del REPEC project.

En casos de duda con algunas citaciones cruzadas también se han empleado como fuentes informativas Medline, the Social Science Citation Index-SSCI (http://thomsonreuters.com/social-sciences-citation-index/) así como la base SCOPUS (http://info.scopus.com/scopus-in-detail/facts/), si bien los criterios de clasificación por palabras clave en cada una de ellas no es el mismo.

Así, por ejemplo, incluso ciñéndonos estrictamente al SSCI y al Journal Citation Report ${ }^{3}$ en su última versión 2012 son seis las publicaciones relacionadas específicamente con Economía de la Salud (a pesar de que también otras publicaciones no específicas de la disciplina publican estudios sobre áreas temáticas de la misma) y que a su vez engloban estudios específicos de eficiencia o de equidad y desigualdades en salud como entre otros:

- Value in Health

${ }^{3}$ Anualmente, Thomson ISI elabora y publica el Informe de Citas (Journal Citation Reports). Esta herramienta es utilizada para la evaluación de las revistas existiendo dos ediciones: JCR Science Edition (para revistas de ciencias) y JCR Social Sciences Edition (para revistas de ciencias sociales). Es en esta última edición donde está incluido y podemos consultar el correspondiente ranking por categorías. Así, en la actualidad, existen 82 revistas incluidas en el área de Ciencias de la Salud y Servicios (Health Care Sciences \& Services). 
- Health Economics

- Pharmacoeconomics

- Journal of Health Economics

- The European Journal of Health Economics

- The International Journal of Health Care Finance and Economics.

\section{Tabla 1}

Top 10 de publicaciones más citadas a contar desde el año 2000 en Economía de la Salud: estudios de eficiencia y equidad

\begin{tabular}{|c|c|c|c|c|c|}
\hline CITAS & AUTORES & AÑO & TITULO & JOURNAL / BOOK & RANGO \\
\hline 934 & $\begin{array}{l}\text { Case, A.C.; Lubotsky, } \\
\text { D. y Paxson, C. }\end{array}$ & 2002 & $\begin{array}{l}\text { Economic Status and Health } \\
\text { in childhood: the origins of } \\
\text { the gradient }\end{array}$ & $\begin{array}{l}\text { American Economic } \\
\text { Review }\end{array}$ & 1 \\
\hline 788 & Le Grand, E & 2003 & $\begin{array}{l}\text { Motivation, agency, and } \\
\text { public policy; Of knights and } \\
\text { knaves, pawns and queens }\end{array}$ & Oxford University Press & 2 \\
\hline 612 & $\begin{array}{l}\text { Kakwani, N., Wagstaff, } \\
\text { A. y Van Doorslaer, E. }\end{array}$ & 1997 & $\begin{array}{l}\text { Socioeconomic inequalities in } \\
\text { health: measurement, } \\
\text { computation, and statistical } \\
\text { inference }\end{array}$ & $\begin{array}{l}\text { Journal of } \\
\text { Econometrics }\end{array}$ & 3 \\
\hline 608 & $\begin{array}{l}\text { Deaton, A.S. y Dreze, } \\
\text { J. }\end{array}$ & 2002 & $\begin{array}{l}\text { Poverty and inequality in } \\
\text { India: A Re-examination }\end{array}$ & $\begin{array}{l}\text { Indian Economy since } \\
\text { independence. } \\
\text { Economic and Political } \\
\text { Weekly }\end{array}$ & 4 \\
\hline 586 & Sen, A.K. & 1998 & $\begin{array}{l}\text { Mortality as indicator of } \\
\text { economic success and failure }\end{array}$ & Economic Journal & 5 \\
\hline 555 & $\begin{array}{l}\text { Van Doorslaer, E., } \\
\text { Wagstaff, A., } \\
\text { Bleichrodt, H., et al. }\end{array}$ & 1997 & $\begin{array}{l}\text { Income-related inequalities in } \\
\text { health: some international } \\
\text { comparisons }\end{array}$ & $\begin{array}{l}\text { Journal of Health } \\
\text { Economics }\end{array}$ & 6 \\
\hline 491 & Williams, A. & 1997 & $\begin{array}{l}\text { Intergenerational equity: an } \\
\text { exploration of the "fair } \\
\text { innings" argument }\end{array}$ & Health Economics & 7 \\
\hline 451 & $\begin{array}{l}\text { Culyer, A.J. y } \\
\text { Wagstaff, A. }\end{array}$ & 1993 & $\begin{array}{l}\text { Equity and equality in health } \\
\text { and health care }\end{array}$ & $\begin{array}{l}\text { Journal of Health } \\
\text { Economics }\end{array}$ & 8 \\
\hline 440 & $\begin{array}{l}\text { Becker, G.S., } \\
\text { Philipson, T.J. y } \\
\text { Soares, R.R. }\end{array}$ & 2005 & $\begin{array}{l}\text { The quantity and quality of } \\
\text { life and the evolution of world } \\
\text { inequality }\end{array}$ & $\begin{array}{l}\text { American Economic } \\
\text { Review }\end{array}$ & 9 \\
\hline 410 & $\begin{array}{l}\text { Van Doorslaer, E., } \\
\text { Wagstaff, A., Van Der } \\
\text { Burg, H., et al. }\end{array}$ & 2000 & $\begin{array}{l}\text { Equity in the delivery of } \\
\text { health care in Europe and the } \\
\text { US }\end{array}$ & $\begin{array}{l}\text { Journal of Health } \\
\text { Economics }\end{array}$ & 10 \\
\hline
\end{tabular}

Fuente: Elaboración propia a partir de las fuentes indicadas anteriormente.

Igualmente, dado que EconLit no tiene exactamente un sistema de citaciones similares a las de las fuentes señaladas se ha utilizado también en algunos casos como fuente de control al Google Scholar (http://scholar.google.es/) donde sí se graban el número de citaciones y además tiene la ventaja que no sólo refleja citaciones en revistas, sino también en libros, capítulos de libros, journals de libre-acceso o restringido, informes técnicos, etc. Para asignar en este caso los 
artículos a esta temática se han tenido en cuenta no sólo el título sino también el abstract y en su caso el contenido del propio artículo especialmente si es accesible via pdf en Google Scholar.

Como resultado puede observarse que los trabajos más importantes publicados en esta temática se refieren especialmente a contribuciones metodológicas novedosas y estudios aplicados incluyéndose comparaciones internacionales (destacando en este último caso las relativas a desigualdades entre distintos países publicadas en 1997 y 2000 en el Journal of Health Economics por Van Doorslaer et al.).

\section{A MODO DE CONCLUSIONES: UNA AGENDA DE FUTURO EN ECONOMÍA DE LA SALUD}

Una hoja de ruta para la investigación futura en Economía de la Salud que pretenda atender a la mejora en eficiencia, equidad y desigualdades de salud debería primar preferentemente aquellos estudios que cumplan una o las dos condiciones siguientes:

- Que impliquen innovaciones metodológicas para las organizaciones sanitarias. Para ello, es básico que puedan ser capaces de traducir los resultados obtenidos en términos de política sanitaria.

- Que actualicen resultados de estudios anteriores al disponer de nuevas bases de datos o información más detallada.

Sin duda, en la agenda de futuro en Economía de la salud, y que dará lugar a numerosas investigaciones, aparecerán temas de gran relevancia como la sostenibilidad en el futuro de los sistemas sanitarios, la revisión de su financiación y las desigualdades territoriales, el copago de determinados servicios sanitarios, la definición de las carteras de servicios básicos, el cambio en estilos de vida, el uso eficiente de las tecnologías de la información (desarrollo de la e-health), la evaluación de las políticas públicas, etc. Elementos todos ellos que contribuirán a mejorar la eficiencia y calidad de la atención sanitaria así como la equidad en el acceso de sus ciudadanos y que al mismo tiempo permitirán reducir las desigualdades en salud.

\section{REFERENCIAS BIBLIOGRAFICAS}

ABADIE, A. y IMBENS, G. (2006): "Large Sample Properties of Matching Estimators for Average Treatment Effects". Econometrica 74(1), 235-267.

ATKINSON, A. (1970): "On the Measurement of Inequality", Journal of Economic Theory, 2, 244-263. 
BECK, K., ZWEIFEL, P., TROTTMANN, M. (2010): "Risk adjustment in health insurance and its long-term effectiveness". Journal of Health Economics, 29(4), 489-498.

BECKER, G., PHILIPSON, T., SOARES, R.R. (2005): "The quantity and quality of life and the evolution of world inequality", American Economic Review, 95(1):277-291.

CABASES, J.M. y ERREA, M. (2010): "Attitudes towards blood and living organ donations", Documentos de Trabajo de la Universidad Pública de Navarra, $\mathrm{n}^{\circ} 1004$.

CANTARERO, D. (2003): "Análisis del gasto sanitario autonómico y su nueva financiación en España”. Investigaciones,19, Instituto de Estudios Fiscales, Madrid.

CASE, A., LUBOTSKY, D., PAXSON, C. (2002): " Economic status and health in childhood: The origins of the gradient", American Economic Review, 92, 1308-1334.

COELLI, T., PRASADA RAO, D.S.y BATTESE, G.E.(1998): An Introduction to Efficiency and Productivity Analysis, Kluwer Academic Publishers, Boston.

COSTA, J. y GIL, J. (2008): "Una Exploración de las Desigualdades Socioeconómicas en Morbilidad en España", Cuadernos Económicos del ICE, 75, 4362.

CULYER, A.J: y WAGSTAFF, A. (1993): "Equity and equality in health and health care", Journal of Health Economics, 12(4), 431-457.

DEATON, A., DREZE, J. (2002): "Poverty and inequality in India: A re-examination", Economic and Political Weekly, 7 September: 3729-3748.

FARRELL, M.J. (1957): "The measurement of productive efficiency", Journal of the Royal Statistical Society Series A,120, 253-290.

FUCHS, V. (2000): "The future of Health Economics". Journal of Health Economics, 19, 141-157.

GINI, C. (1921): "Measurement of Inequality of Incomes", The Economic Journal, 31, 124-126.

GROSSMAN M. (1972): The Demand for Health: A Theoretical and Empirical Investigation.MA, NBER, Cambridge.

HECKMAN J. (2001): "Micro Data, Heterogeneity, and the Evaluation of Public Policy: Nobel Lecture", Journal of Political Economy Vol. 109, n. ${ }^{\circ} 4$, pp. 673748.

HECKMAN, J., ICHIMURA, H. y TODD, P. (1998): "Matching as an Econometric Evaluation Estimator", The Review of Economic Studies 65(2), pp. 261-294.

HOLLINGSWORTH, B. (2008): "The Measurement of Efficiency and Productivity of Health Care Delivery", Health Economics, 17(10):1107-28.

HOLLINGSWORTH, B., STREET, A. (2006): "The market for efficiency analysis of health care organisations", Health Economics, 15(10), 1055-1059. 
HUA, H., LI-XIN, S., XIAN-LI, Z., SHENG-XIN, C. (2009): "Data envelopment analysis-based evaluation of pharmacy efficiencies of military hospitals", Academic Journal of Second Military Medical Universitary 30 (5), 553-557.

IMBENS, G. W. y WOOLDRIDGE, J. M. (2009): "Recent Developments in the Econometrics of Program Evaluation", Journal of Economic Literature 47, pp. 5-86.

JAKOBSSON, U. (1976): "On the Measurement of the Degree of Progression", Journal of Public Economics, 5, 161-168.

KAKWANI, N. C. (1977): "Applications of Lorenz Curves in Economic Analysis", Econometrica, vol.45, 719-727.

KAKWANI, N.C. (1980). Income Inequality and Poverty. Methods of Estimation and Policy Implications. Oxford University Press, Oxford.

KAKWANI, N.C., WAGSTAFF, A., VAN DOORSLAER, E., (1997): "Socioeconomic inequalities in health: Measurement, computation and statistical inference", Journal of Econometrics, 77(1): p. 87-104.

$\mathrm{KAO}$, L.; LU, CH. y CHI, CH. (2011): "Efficiency measurement using independent component analysis and data envelopment analysis", European Journal of Operational Research, 210 (2), 310-317.

KOLM, S. (1968): "The Optimal Production of Social Justice", en J. MARGOLIS y H. GUITTON, (eds.) Public Economics, MacMillan.

KOLM, S. (1976): "Unequal Inequalities", Journal of Economic Theory, 12, 416422.

LABELLE, R., STODDART, G., RICE, T. (1994): "A re-examination of the meaning and importance of supplier-induced demand". Journal of Health Economics, 13(3),347-368.

LE GRAND, J. (2003): Motivation, agency, and public policy; Of knights and knaves, pawns and queens. Oxford University Press.

LELEU, H., MOISES, J., VALDAMANIS, V. (2012): "Optimal productive size of hospital's intensive care units", International Journal of Production Economics, 136(2), 297-305.

LEY, E. (1991). Eficiencia productiva: un estudio aplicado al sector hospitalario, Investigaciones Económicas, 15,1:71-88.

LOPEZ-CASASNOVAS G. y WAGSTAFF, A. (1988): "La combinación de los factores productivos en el hospital: Una aproximación a la función de producción", Investigaciones Económicas 1988; XII: 2:305-327.

LOVELL, C.A.K. (1993): "Production Frontiers and Productive Efficiency". En FRIED, H.O, LOVELL, C.A.K. y SCHMIDT, S.S. Schmidt (eds.), The measurement of Productive Efficiency: Techniques and Applications, Oxford University Press, Oxford: 3-67.

MACKENBACH, J.P. (2005): «Health Inequalities: Europe in Profile», Informe independiente comisionado y publicado por la presidencia del Reino Unido de la UE. Disponible en: http://www.fco.gov.uk/Files/kfile/HI_EU_Profile,0.pdf. 
MARTIN, J.J. y PUERTO, M. (2007): "La medida de la eficiencia en las organizaciones sanitarias". Presupuesto y gasto público, 49, 139-161.

MONTERO, R. y JIMENEZ, J.D. (2008): "Evolución de la equidad interterritorial en el acceso a los servicios sanitarios entre las CCAA españolas (19872003) y su relación con la evolución del gasto sanitario público", Cuadernos Económicos del ICE, 75, 167-190.

MURILLO, C. y GONZALEZ, B. (2006): "Limitaciones y potencialidades de las ligas de calidad de los proveedores sanitarios". Estudios de Economía Aplicada, 24, 777-788.

NEWHOUSE, J.P. (1970): "Toward a Theory of Nonprofit Institutions: An Economic Model of a Hospital". American Economic Review, 60(1), 64-74.

PASCUAL, M. y CANTARERO, D. (2009): "Intergenerational health mobility: an empirical approach based on the ECHP" Applied Economics, 41(4), 451458.

PEIRO, S. (2006): «Algunas reflexiones sobre la organización de la información sanitaria en el Sistema Nacional de Salud», Revista de Administración Sanitaria, 4 (I): 81-94.

PHELPS, C. (1986): "Induced Demad - Can We Ever Know Its Extent?". Journal of Health Economics, 5(4), 355-365.

PUIG-JUNOY, J. (2000): "Partitioning input cost efficiency into its allocative and technical components: An empirical DEA application to hospitals", Socioeconomic Planning Sciences, 34(3), 99-218.

PUIG-JUNOY, J. y DALMAU, E. (2000): «¿Qué sabemos acerca de la eficiencia de las organizaciones sanitarias en España? Una revisión de la literatura económica», en Avances en la gestión sanitaria: Implicaciones para la política, las organizaciones sanitarias y la práctica clínica, XX Jornadas de Economía de la Salud, Asociación de Economía de la Salud, Barcelona.

RIVERA, B., CURRAIS, L., RUNGO, P. (2008): "La transmisión de las desigualdades en el estado de salud: Efectos sobre la equidad intergeneracional a partir de la acumulación de capital humano", Cuadernos Económicos del ICE, 75, 99-114.

ROSENBAUM, P. y RUBIN, D. (1983): "The Central Role of the Propensity Score in Observational Studies for Causal Effects", Biometrika 70(1), 41-55.

RUBIN, R.M. y CHANG, C.F. (2003): "A bibliometric analysis of health economics articles in the economics literature: 1991-2000", Health Economics, 12, 403-414.

SEN, A. (1973): On Economic Inequality. Oxford Clarendon Press y New York Norton.

SEN, A. (1992): Inequality Reexamined. Harvard University Press. Cambridge, MA, Clarendon Press: Oxford.

SEN, A. (1998): "Mortality as an indicator of economic success and failure", The Economic Journal, Vol. 108(446), 1-25. 
STIGLITZ, J. (1994): "Replanteamiento del papel del Estado en la economía. La provisión de bienes privados suministrados públicamente". En LOPEZ I CASASNOVAS, G. (dir.), Análisis económico de la sanidad, Barcelona.

STOYANOVA, A., RODRIGUEZ, M. y PINILLA, J. (2008): "El mapa de las desigualdades en salud por grupos de edad en España", Cuadernos Económicos del ICE, 75, 21-42.

THOUEZ, J.P. (2006): "The influence of poverty, deprivation and social class on health inequality: a review of American and Canadian literature". Estudios de Economia Aplicada, 24, 755-776

VAN DOORSLAER, E., GERDTHAM, U.-G. (2003): «Does inequality in selfassessed health predict inequality in survival by income», Social Science and Medicine, 57, 1621-1629.

VAN DOORSLAER, E., WAGSTAFF, A., BLEICHRODT, H., et al. (1997): "Income-related inequalities in health: some international comparisons", Journal of Health Economics 16, 93-112.

VAN DOORSLAER, E., WAGSTAFF, A., VAN DER BURG., H. et al. (2000): " Equity in the delivery of health care in Europe and the US ", Journal of Health Economics, 19(5), 553-583.

WAGSTAFF, A. (1989): "Estimating efficiency in the hospital sector: a comparison of three statistical cost frontier models", Applied Economics, 21, 659672.

WAGSTAFF, A. y CULYER, A.J. (2012): "Four decades of health economics through a bibliometric lens". Journal of Health Economics, 31, 406-439.

WAGSTAFF, A. y VAN DOORSLAER, E (2000): «Equity in Health Care Finance and Delivery», en Culyer, A.J y Newhouse, J.P (ed.). Handbook of Health Economics, Cap. 34, Elsevier Science, B.V.

WILKINSON, R. (1997): «Health Inequalities: Relative or Absolute Material Standards», British Medical Journal, 314(7080), 591-95.

WILLIAMS, A. (1997) "Intergenerational equity: An exploration of the 'fair innings' argument", Health Economics. 6(2):117-32.

WORLD HEALTH ORGANIZATION (2009). Resolution EB124/R6 on Reducing health inequities through action on the social determinants of health. Geneva, Executive Board. WHO (http://apps.who.int/gb/ebwha/pdf_files/EB124/ B124_R6-en.pdf, [accessed 29 January 2010]).

ZEWIFEL, P. (2013): "The present state of health economics: a critique and an agenda for the future". European Journal of Health Economics, 14, 569-571. 